\title{
Fast Substrate Noise-Aware Floorplanning with Preference Directed Graph for Mixed-Signal SOCs
}

\author{
Minsik Cho, Hongjoong Shin and David Z. Pan \\ Dept. of ECE, The University of Texas at Austin, Austin, TX 78712 \\ thyeros@cerc.utexas.edu, unishin@cerc.utexas.edu,dpan@ece.utexas.edu
}

\begin{abstract}
In this paper, we introduce a novel substrate noise estimation technique during early floorplanning, based on the concept of Block Preference Directed Graph (BPDG) and the classic Sequence Pair (SP) floorplan representation. Given a set of analog and digital blocks, the BPDG is constructed based on their inherent noise characteristics to capture their preferred relative orders for substrate noise minimization. For each sequence pair generated during floorplanning evaluation, we can measure its violation against BPDG very efficiently. We observe that by simply counting the number of violations obtained in this manner, it correlates remarkably well with accurate but computationintensive substrate noise modeling. Thus, our BPDG-based model has high fidelity to guide the substrate noise-aware floorplanning and layout optimization, which become a growing concern for mixed-signal/RF system on chips (SOC). Our experimental results show that the proposed approach is over 60x faster than conventional floorplanning with even very compact substrate noise models. We also obtain less area and total substrate noise than the conventional approach.
\end{abstract}

\section{INTRODUCTION}

Continuing demand for data and telecommunication application is driving tighter integration of many heterogeneous functions into a single system-on-chip. These components can be pre-designed IP cores of different natures such as sensitive front-end RF circuits, high-precision analog/mixed-signal circuits, and high-performance digital circuits. Therefore, the interference between these heterogeneous components has to be considered during layout planning and optimization [1]. A key interference is the substrate noise caused by large amount of switching activities in high speed digital cores, to the analog/RF components. It may degrade the reliability and performance of these sensitive analog/mixed-signal/RF IPs [2]. The problem is becoming a growing concern due to higher clock frequency, more accurate analog precision, deeper technology scaling, and the integration of front-end RF with digital blocks [3]-[5]. Many effects that corrupt RF signal such as DC offset, oscillator pulling and pushing, local oscillator leakage can be traced to the substrate-coupled noise [2].

Therefore, fast yet accurate evaluation and optimization of substrate noise in physical design has become a crucial part of mixed-signal SOC designs, in order to avoid expensive overdesign and excessive design iterations. A key step of such layout optimization is the floorplanning stage [6]. Although

This work is supported in part by SRC under contract 2005-TJ-1321, IBM Faculty Award, and equipment donations from Intel. many works have been done in modeling and simulation of substrate noise [2], [3], [5], [12]-[15], there is not much in the literature on substrate noise optimization in early floorplanning stage. Lin et al. [7] proposed an optimization technique that incorporates substrate noise minimization into optimization loops. This technique, however, requires detailed and expensive circuit simulations to estimate the coupled substrate noise. Blakiewicz et al. [8] proposed a floorplanning algorithm with substrate noise as a cost function. A more scalable substrate model with frequency-dependent sensitivity function of analog and digital blocks is used, but still it requires significant computational overhead to evaluate the substrate noise cost function during floorplanning.

In this paper, we propose a novel concept of block preference directed graph (BPDG) to overcome the modeling bottleneck for substrate noise-aware floorplanning. Our BPDGbased model has high fidelity compared with accurate but much more expensive substrate noise modeling. Thus, it is suitable to guide MS-SOC floorplanning. The major contributions of this paper include the following.

- We introduce the novel concept of block preference directed graph (BPDG) to represent the preferred relative block locations in floorplanning. In BPDG, all the preferences are decided to minimize the substrate noise, and each preference is specified as a directed edge.

- We propose a fast substrate noise estimation algorithm by combining BPDG and sequence pair. We simply count how many preferences in BPDG are not held in a sequence pair with simple bitwise-OR operation.

- We show that our approach has surprisingly high fidelity to the substrate noise calculated by a most recent, accurate substrate noise model [5].

- We propose a fast substrate noise-aware floorplanning algorithm with BPDG and sequence pair. Our experimental results show the proposed approach is significantly (at least 60x) faster than a conventional simulation-based, substrate noise-aware floorplanning.

The rest of the paper is organized as follows. In Section II, preliminaries are described. In Section III, the concept of block preference directed graph is introduced. The fast substrate noise estimation algorithm is proposed in Section IV, and the overall floorplanning flow is described in Section V. Experimental results are discussed in Section VI. Section VII concludes this paper with future work. 


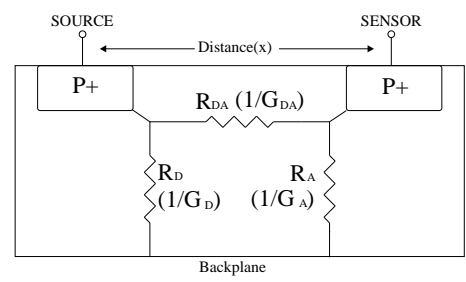

Fig. 1. Macromodel for the substrate

\section{PRELIMINARIES}

\section{A. Sequence Pair and Block Alignment}

A sequence pair [9] is a pair of sequences of $n$ elements representing a list of $n$ blocks. Two sequences specify the geometric relations between each pair of blocks. For example, (..A..B...,.A..B..) means that a block $\mathrm{A}$ is to the left of a block B, and (..B..A.., ..A..B..) implies that A is below B. A sequence pair can be translated into a floorplan by horizontal and vertical constraint graph [9]. Conditions for block alignments in sequence pair are studied in [10]. H/V alignment constraints and abutting constraint between blocks are introduced and applied for performance-aware floorplanning.

\section{B. Substrate Noise Model}

Several techniques have been proposed to model and analyze substrate noise accurately in integrated circuit level [11][13]. However, these techniques require the detailed implementation information in transistors and time-intensive transistor-level simulation. In this paper, we use compact substrate coupling model [5] to evaluate an instance of floorplan from conventional approach, and to verify the final floorplan. The model in [5] is known to be highly scalable with dimensions and separations.

A two-port lumped resistor network, modeling substrate is illustrated in Fig. 1. The resistance $R_{D A}$, models the coupling between two blocks, and $R_{A}$ and $R_{D}$ model the coupling from the blocks to the backplane. The resistances, $R_{D A}, R_{A}$ and $R_{D}$ can be derived from the scalable macromodel, which is based on Z-parameters.

$$
Z=\left[\begin{array}{cc}
Z_{11} & Z_{12} \\
Z_{21} & Z_{22}
\end{array}\right]=\frac{1}{\triangle}\left[\begin{array}{cc}
G_{D}+G_{D A} & G_{D A} \\
G_{D A} & G_{A}+G_{D A}
\end{array}\right]
$$

where $\triangle=G_{A} G_{D A}+G_{D} G_{D A}+G_{A} G_{D}$ and any $Z_{i j}$ can be calculated with equations in [5], [14], [15].

The coupling gain (propagation gain) of the substrate can be calculated from the value of resistors in the two-port lumped network shown in Fig. 1. The coupling gain of $i$-th digital block to $j$-th analog block, $C G_{i, j}$ is given by:

$$
C G_{i, j}=\frac{R_{A}}{R_{A}+R_{D A}}=\frac{G_{D A}}{G_{D A}+G_{A}}=\frac{Z_{12}}{Z_{22}}
$$

Although $C G_{i, j}$ exhibits frequency-dependent characteristics, it is constant under a few gigahertz [4]. In this paper, we assume that the bands of interest are within this limit. The quantity of the substrate noise can be estimated using frequency-dependent characteristics of noise source and sensor block, and a simple analytical formula based on $C G_{i, j}$. The substrate noise of $j$-th analog block from switching of $i$-th digital block, $N_{i, j}$ can be approximated by [8]:

$$
N_{i, j}=\left(C G_{i, j}\right) \cdot \sqrt{\int_{0}^{\infty}\left(S_{i}(f) \cdot H_{j}(f)\right)^{2} d f}
$$

where $S_{i}(f)$ and $H_{j}(f)$ are Power Spectral Density (PSD) of noise source and transfer function of noise sensor respectively. Also, the total noise from all digital blocks is:

$$
N_{\text {total }}=\sum_{i} \sum_{j} N_{i, j}
$$

As shown in Eqn. (3), $C G_{i, j}$ is scaled by average power of noise with regard to the frequency. The frequency-dependent noise generated by a digital block, $S_{i}(f)$ is shaped by the transfer function of the noise sensor. The integration of the shaped power of noise represents the quantity of noise injected into analog block, when $C G_{i, j}$ is equal to 1 . We use a piecewise-linear approximation of PSD to estimate $S_{i}(f)$, and Power/Ground bounce limits to determine its parameters.

\section{BPDG: BLOCK PREFERENCE DiRECTED GRAPH}

The substrate noise model in Section II-B is one of the most compact models with high scalability and accuracy. However, it is still computationally expensive to perform a substrate noise estimation even with such an efficient model during simulated annealing-based floorplanning, because every noise estimation after a movement requires the accurate location of every block (substrate noise is exponentially sensitive to geometric distance [5], [14], [15]), whereas area and wirelength can be calculated approximately. Furthermore, computing noise itself with Eqn. $(2,3)$ is not computationally trivial.

For fast substrate noise estimation, a new concept of block preference directed graph, BPDG is introduced and described in this section. BPDG represents preferred relative locations of blocks to guide substrate noise-aware floorplanning. BPDG construction consists of three steps.

1) A table of substrate noise between all analog and digital blocks is constructed.

2) Analog block orderings and digital block orderings are created separately with the substrate noise table.

3) BPDG is constructed by finding common orders from block orderings.

The following subsections illustrate each step with detailed examples in Table I and Fig. 2, 3, 4.

\section{A. Substrate Noise Table Construction}

Since substrate noise is heavily related to the distance between blocks, we assume that the nominal distance is fixed to normalize the effect of distance. With such fixed distance, the substrate noise between a digital block and an analog block purely depends on frequency coupling and geometric properties like area and perimeter [5], [14], [15]. Under such condition, for each digital block $D_{i}$ and analog block $A_{j}$, a substrate noise on $A_{j}$ due to $D_{i}, N_{i j}$ can be computed from Eqn. (3). Table I shows an example of substrate noise table of between digital blocks $\left(D_{1}, D_{2}, D_{3}, D_{4}, D_{5}, D_{6}\right)$ and analog blocks $\left(A_{1}, A_{2}, A_{3}\right)$. 
TABLE I

SubSTRATE NOISE TABLE

\begin{tabular}{|c|c|c|c|c|c|c|}
\hline & $D_{1}$ & $D_{2}$ & $D_{3}$ & $D_{4}$ & $D_{5}$ & $D_{6}$ \\
\hline$A_{1}$ & 5 & 2 & 6 & 3 & 10 & 1 \\
$A_{2}$ & 2 & 1 & 3 & 10 & 8 & 5 \\
$A_{3}$ & 3 & 8 & 7 & 11 & 9 & 12 \\
\hline
\end{tabular}

\section{B. Analog Block Ordering}

Based on the substrate noise table, analog blocks can be sorted for each digital block by the descending order of substrate noise. Consider the example in Table I. Analog block $A_{1}, A_{3}$ and $A_{2}$ can be ordered by the substrate noise from $D_{1}$, as $N_{11}=5>N_{13}=3>N_{12}=2 . D_{1}-A_{1}$ is $5, D_{1}-A_{2}$ is 2 and $D_{1}-A_{3}$ is 3 . The other five orderings can be obtained in the same manner, as shown in Fig. 2. Basically, this ordering pushes more noise-sensitive analog blocks to the head and less sensitive ones to the tail of block orderings.

$$
\begin{array}{ll}
D_{1}: A_{1} \leftarrow A_{3} \leftarrow A_{2} & , \quad D_{2}: A_{3} \leftarrow A_{1} \leftarrow A_{2} \\
D_{3}: A_{3} \leftarrow A_{1} \leftarrow A_{2} & , \quad D_{4}: A_{3} \leftarrow A_{2} \leftarrow A_{1} \\
D_{5}: A_{1} \leftarrow A_{3} \leftarrow A_{2} & , \quad D_{6}: A_{3} \leftarrow A_{2} \leftarrow A_{1}
\end{array}
$$

Fig. 2. Analog block orderings

\section{Digital Block Ordering}

In similar way, digital blocks can be sorted for each analog block by ascending order of substrate noise. Again considering the example in Table I, digital block $D_{6}, D_{2}, D_{4}, D_{1}, D_{3}$ and $D_{5}$ can be ordered such that the substrate noise on $A_{1}$ is increasing. All digital block orderings are shown in Fig. 3. This pushes less aggressive blocks to the head and more aggressive blocks to the tail of block orderings.

$$
\begin{array}{ll}
A_{1}: & D_{6} \leftarrow D_{2} \leftarrow D_{4} \leftarrow D_{1} \leftarrow D_{3} \leftarrow D_{5} \\
A_{2}: & D_{2} \leftarrow D_{1} \leftarrow D_{3} \leftarrow D_{6} \leftarrow D_{5} \leftarrow D_{4} \\
A_{3}: & D_{1} \leftarrow D_{3} \leftarrow D_{2} \leftarrow D_{5} \leftarrow D_{4} \leftarrow D_{6}
\end{array}
$$

Fig. 3. Digital block orderings

\section{BPDG Construction}

The two key ideas behind BPDG construction are: first, to find common block order patterns in order to minimize the substrate noise; second, to make less aggressive digital blocks and less sensitive analog blocks interfaced. An analog BPDG and a digital BPDG are constructed with analog and digital block orderings by Algorithm 1. The reason to create a virtual vertex in Algorithm 1 is to force analog blocks isolated from digital blocks, which is common in real mixed-signal design.

Consider the final BPDG in Fig. 4 as an example. Since $A_{3}$ is before $A_{2}$ for all analog block orderings in Fig. 2, vertices $A_{3}$ and $A_{2}$ are inserted into $G_{a}$ (Analog BPDG), and connected with a directed edge. Again, vertices $D_{1}$ and $D_{3}$

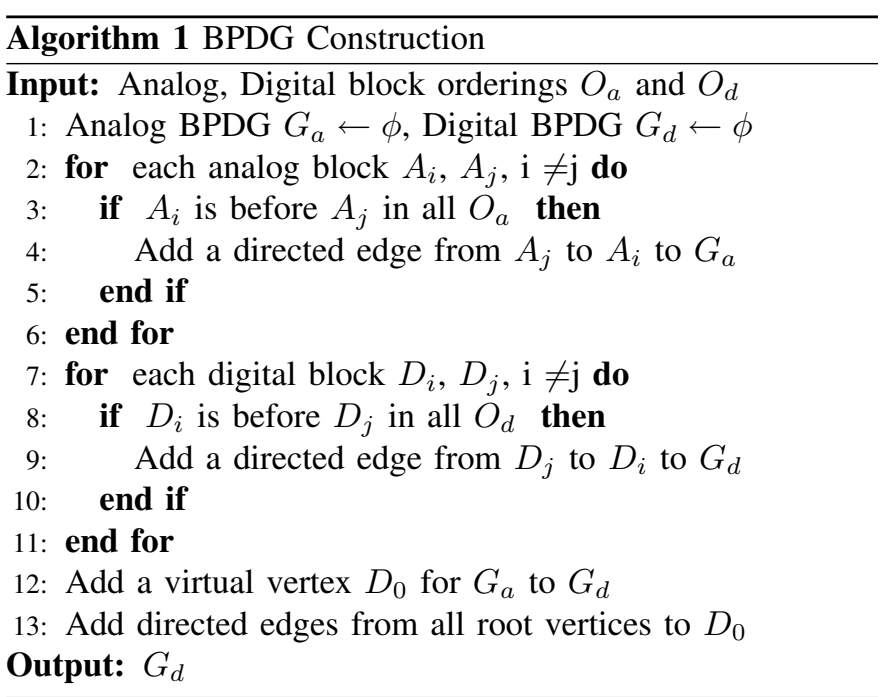

are inserted into $G_{d}$ (Digital BPDG) with a directed edge from $D_{3}$ to $D_{1}$, as $D_{1}$ is before $D_{3}$ for all digital block orderings in Fig. 3. Note that $A_{1}$ does not have any edge, as there is no common order regarding $A_{1}$ in Fig. 2, and $D_{6}$ only has an edge to $D_{0}$ for analog-digital separation. Lastly, $G_{a}$ and $G_{d}$ are merged via virtual vertex $D_{0}$.

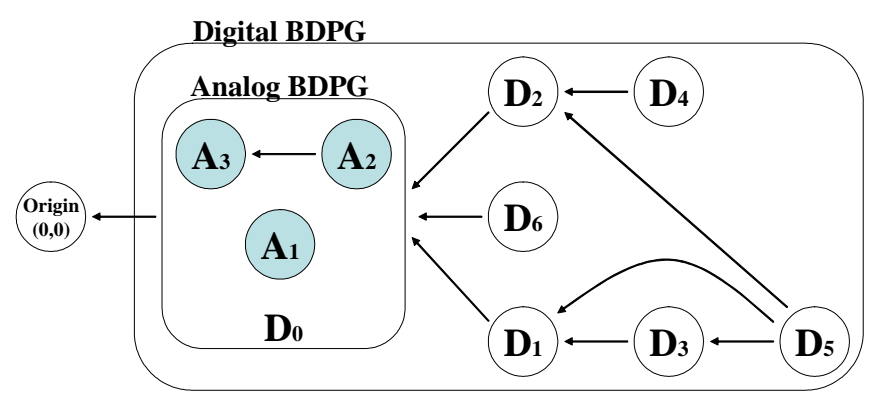

Fig. 4. Block preference directed graph (BPDG)

\section{Substrate Noise Estimation with BPDG}

The BPDG in Section III can be used to estimate substrate noise quickly by comparing it against a sequence pair which is one of the most popular floorplan representations. In this section, a theorem which returns the number of violations against preferences in a BPDG from a sequence pair is presented, and its high fidelity to substrate noise is shown. The number of violations is highly correlated to substrate noise quantity; intuitively, more violations indicate more noise, because each directed edge from a block $B_{a}$ to a block $B_{b}$ in the BPDG means that $B_{b}$ is preferred to be closer to the origin (left-bottom corner of floorplan) than $B_{a}$ to reduce the substrate noise.

\section{A. Sequence Pair with BPDG}

In [10], the concept of strictly ahead is defined for block alignment in a floorplanning with sequence pair. When there is no block between $B_{a}$ and $B_{b}$ in a floorplan, $B_{a}$ is strictly 
ahead of $B_{b}$. Fig. 5(a) shows a floorplan with several blocks. In this example, $B_{a}$ is strictly ahead of $B_{1}, B_{2}, B_{3}$ and $B_{4}$. In fact, strictly ahead is a necessary condition for two blocks to be abutted (only $B_{1}$ and $B_{3}$ are abutted to $B_{a}$ ). In the following, we introduce several definitions based on strictly ahead for easier explanation of this section.

Definition $\alpha$. Given a block $B_{a}$ and a sequence pair $(\mathrm{P}, \mathrm{N})$, all the blocks which are both strictly ahead of $B_{a}$ and below $B_{a}$ form a strictly below set of $B_{a}$.

DEFINITION $\beta$. Given a block $B_{a}$ and a sequence pair $(\mathrm{P}, \mathrm{N})$, all the blocks which are both strictly ahead of $B_{a}$ and to the left of $B_{a}$ form a strictly left set of $B_{a}$.

DEFINITION $\gamma$. Given a block $B_{a}$ and a sequence pair $(\mathrm{P}, \mathrm{N})$, any block in a strictly below/left set of $B_{a}$ and abutting to $B_{a}$ is a reference block.

In Fig. 5(a), $B_{2}, B_{3}$ and $B_{4}$ are strictly below set of $B_{a}$, because they are strictly ahead of $B_{a}$ as well as below $B_{a}$. Also, $B_{3}$ is a reference block of $B_{a}$. One intuitive property of the reference block is stated in Lemma $\alpha$ referring to [10].

Lemma $\alpha$ : If a block $B_{a}$ has a strictly below/left set $S$, there must exist some reference block $B_{x}$ in $S$ under a completely packed floorplan.

Based on Lemma $\alpha$, the relative locations of two blocks can be determined. Consider a specific floorplan in Fig. 5(b) where $B_{a}$ is to the left of $B_{b}$, and $B_{x}$ is a reference block of $B_{a}$. It can be easily proved that if a block such as $B_{x}$ exists below $B_{b}$, it is guaranteed that $B_{a}$ has a shorter distance to the origin $(0,0)$ than $B_{b}$. This key idea to compare the relative location of two blocks conservatively with a sequence pair is presented as Theorem 1 by extending Theorem 1 and 3 in [10]. Note that the conditions 1) and 2) of Theorem 1 are corresponding to Fig. 5(b) and (c) respectively.

Theorem 1: Let $S_{b}$ be a strictly below set of $B_{a}$ and $S_{l}$ a strictly left set of $B_{a}$. A block $B_{a}$ is guaranteed to have shorter distance to the left bottom corner than a block $B_{b}$ under a completely packed floorplan, if either of following conditions is satisfied.

1) for any block $B_{s}$ in $S_{b}$, a sequence pair $(P, N)$ is (.. $\left.B_{a} X_{1} B_{b} X_{2} B_{s} . ., . . B_{s} Y_{1} B_{a} . . B_{b} ..\right)$.

2) for any block $B_{s}$ in $S_{l}$, a sequence pair $(P, N)$ is (.. $\left.B_{s} X_{3} B_{b} X_{4} B_{a} . ., . . B_{s} Y_{2} B_{a} . . B_{b} ..\right)$.

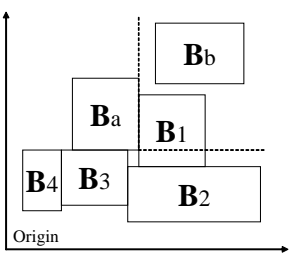

(a)

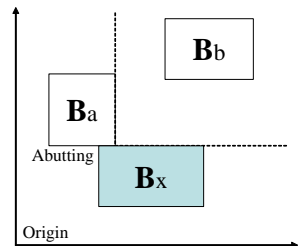

(b)

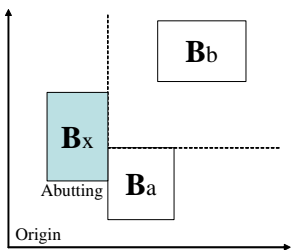

(c)
Fig. 5. Floorplan examples
Thus, when a sequence pair $(P, N)$ and a BPDG $G$ are given, the preferred relative block location (an edge) in $G$ can be examined with Theorem 1 to see if such preference is held in $(P, N)$. Theorem 1 can be further simplified into Theorem 2 with the longest common string $(L C S)$ search by narrowing down the size of subsequences to scan.

Theorem 2: $A$ block $B_{a}$ is guaranteed to have shorter distance to the left-bottom corner than a block $B_{b}$ under a completely packed floorplan, if either of following conditions is satisfied.

1) there is no block $B_{s}$ satisfying $\operatorname{LCS}\left(X_{1}, Y_{1}\right)=\phi$ in a sequence pair $(P, N)=\left(. . B_{a} X_{1} B_{s} . . B_{b} . ., . . B_{s} Y_{1} B_{a} . . B_{b} ..\right)$.

2) there is no block $B_{s}$ satisfying $\operatorname{LCS}\left(X_{2}, Y_{2}\right)=\phi$ in a sequence pair $(P, N)=\left(. . B_{b} . . B_{s} X_{2} B_{a} . ., . . B_{s} Y_{2} B_{a} . . B_{b} ..\right)$.

The following sequence pairs show examples with the BPDG in Fig. 4. Note that the blocks one need to pay attention to are marked with *, and we highlight one violation, even though there can be more.

- $\left(D_{0} D_{6} D_{1} D_{2}^{*} D_{3} D_{4}^{*} D_{5}, D_{4}^{*} D_{0} D_{6} D_{1} D_{2}^{*} D_{3} D_{5}\right)$ This case has $D_{2} \leftarrow D_{4}$ violation, because $D_{2}$ is after $D_{4}$ in the second sequence which does not match either one of required sequence pair patterns in Theorem 1 .

- $\left(D_{4} D_{5} D_{1}^{*} D_{2} D_{6} D_{0}^{*} D_{3}^{*}, D_{0}^{*} D_{5} D_{4} D_{1}^{*} D_{2} D_{6} D_{3}^{*}\right)$

This case has $D_{1} \leftarrow D_{3}$ violation. $D_{0}$ is below $D_{1}$ and $\operatorname{LCS}\left(D_{2} D_{6}, D_{5} D_{4}\right)=\phi$ But, $D_{0}$ is before $D_{3}$ in the first sequence which violates the required sequence pair pattern in condition 1) of Theorem 1.

The significance of Theorem 1 and 2 is that a geometric distance from the origin to any two blocks in a sequence pair can be compared conservatively without other geometric information. Thus, whether an edge (preference) in a BPDG is held in a sequence pair can be checked extremely efficiently. Note that in a real implementation, bitwise-OR can be used instead of $L C S$ computation, since we are only interested in whether there is a common sequence.

\section{B. Fidelity of $B P D G$}

In order to measure the fidelity of BPDG-based model for substrate noise estimation, ami33 from MCNC benchmarks [16] was simulated with carefully generated noise characteristics. Fig. 6 shows the normalized substrate noise on all analog blocks by the number of violations counted by Theorem 2 with different total number of violations. It shows that normalized substrate noise increases near linearly as the

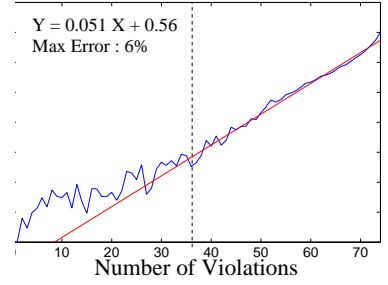

(a) Total 76 violations

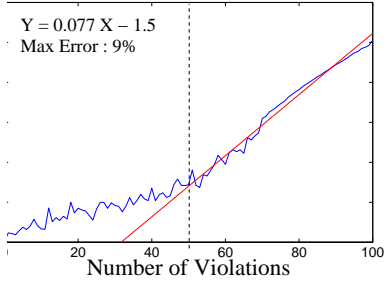

(b) Total 100 violations
Fig. 6. Number of violations vs. Substrate noise 
TABLE II

EXPERIMENTAL RESULTS

\begin{tabular}{|c|c|c|c|c|c|c|c|c|c|}
\hline \multirow[t]{2}{*}{ Name } & \multirow{2}{*}{$\begin{array}{c}\text { Algorithm } \\
\text { Description } \\
\end{array}$} & \multirow{2}{*}{$\begin{array}{c}\text { Cost }^{\mathrm{a}} \\
\text { Function }\end{array}$} & \multirow{2}{*}{$\begin{array}{l}\text { Input } \\
\text { (node) }\end{array}$} & \multirow{2}{*}{$\begin{array}{c}\text { Area } \\
\left(\mathrm{mm}^{2}\right)\end{array}$} & \multirow{2}{*}{$\begin{array}{c}\text { White } \\
\text { Space }(\%)\end{array}$} & \multirow{2}{*}{$\begin{array}{l}\text { Normalized } \\
\text { Noise }\end{array}$} & \multirow{2}{*}{$\begin{array}{l}\text { CPU } \\
(\mathrm{sec})\end{array}$} & \multicolumn{2}{|c|}{ Overhead (\%) } \\
\hline & & & & & & & & $\mathrm{CPU}$ & Area \\
\hline \multirow{4}{*}{ parq } & \multirow{4}{*}{$\begin{array}{c}\text { Pure Parquet } \\
\text { with Sequence Pair }\end{array}$} & \multirow{4}{*}{$\frac{A}{A_{r}}$} & ami33 & 1.19 & 3.2 & 821.1 & 0.8 & 0.0 & 0.0 \\
\hline & & & $\operatorname{ami} 49$ & 36.7 & 3.6 & 1629.9 & 2.6 & 0.0 & 0.0 \\
\hline & & & $\mathrm{n} 75$ & 42.04 & 4.0 & 3559.9 & 8.6 & 0.0 & 0.0 \\
\hline & & & $\mathrm{n} 100$ & 18.86 & 5.1 & 4697.5 & 24.6 & 0.0 & 0.0 \\
\hline \multirow{4}{*}{ bpdg $^{\mathrm{b}}$} & \multirow{4}{*}{$\begin{array}{c}\text { BPDG } \\
\text { with Sequence Pair }\end{array}$} & \multirow{4}{*}{$0.6 \frac{A}{A_{r}}+0.4 \frac{N V}{N V_{r}}$} & ami33 & 1.24 & 6.9 & 121.2 & 0.9 & 15.5 & 3.8 \\
\hline & & & ami49 & 37.9 & 7.1 & 72.2 & 2.7 & 6.6 & 3.4 \\
\hline & & & $\mathrm{n} 75$ & 43.12 & 6.6 & 173.1 & 9.2 & 7.1 & 2.6 \\
\hline & & & $\mathrm{n} 100$ & 19.22 & 6.9 & 202.5 & 26.4 & 7.1 & 1.9 \\
\hline \multirow{4}{*}{ modl } & \multirow{4}{*}{$\begin{array}{l}\text { Substrate Noise Model } \\
\text { with Sequence Pair }\end{array}$} & \multirow{4}{*}{$0.6 \frac{A}{A_{r}}+0.4 \frac{S N}{S N_{r}}$} & ami33 & 1.23 & 6.1 & 143.9 & 73.0 & 8782.5 & 2.8 \\
\hline & & & ami49 & 38.4 & 8.4 & 90.8 & 158.3 & 6103.3 & 4.6 \\
\hline & & & $\mathrm{n} 75$ & 44.08 & 9.1 & 322.4 & 666.9 & 7692.5 & 4.9 \\
\hline & & & $\mathrm{n} 100$ & 19.94 & 11.1 & 696.1 & 1956.3 & 7844.1 & 5.8 \\
\hline
\end{tabular}

a $A, N V$ and $S N$ denote total area, the number of violations and total substrate noise on analog blocks respectively. $A_{r}, N V_{r}$ and $S N_{r}$ are the reference values of $A, N V$ and $S N$ respectively.

$\mathrm{b}$ for bpdg, each side of the virtual analog block is inflated by $0.6 \%$ as a whitespace(guard ring) insertion.

number of violations increases. Notice that the range over $50 \%$ of maximum violations shows high fidelity with less than $6 \%$ error in Fig. 6(a), and 9\% in Fig. 6(b). Since the typical number of violations during simulated annealing falls in this high fidelity range, the number of violations in sequence pair is a good indicator of substrate noise. Thus, by comparing BPDG of Section III against sequence pair, substrate noise can be estimated very fast with high fidelity.

- Our approach estimates substrate noise without accurate geometric information such as $x$ and $y$ coordinates. As a result, all the efforts to compute the accurate locations of all blocks can be saved.

- Our approach needs only integer and bitwise-OR operations whereas model-based noise estimation requires floating point operations and transcendental functions like $\exp (x)$.

\section{Fast Substrate Noise-Aware Floorplanning}

Our floorplanning algorithm takes advantage of the BPDGbased fast substrate noise estimation, efficiently examining discrepancy between BDPG in Section III and sequence pair in Section IV. Also, using block inflation, we insert whitespace around analog blocks as a guard ring. Note that our white space allocation is done as a preprocessing to minimize area overhead. The overall algorithm is described in Algorithm 2.

\section{EXPERIMENTAL RESULTS}

We implemented the proposed algorithm in $\mathrm{C}++$ by modifying Parquet [17] which is a simulated annealing-based floorplanning package available in [18]. In order to compare our approach (bpdg in Table II) with other approaches, we also implemented the conventional model-based, substrate noiseaware floorplanning algorithm (modl in Table II). After every movement inside the simulated annealing loop, to estimate current floorplan instance's substrate noise on the analog blocks, the number of violations by Theorem 2 was counted for bpdg, whereas substrate noise was computed for modl based on the substrate noise model, i.e., Eqn. (4) in Section II-B. The

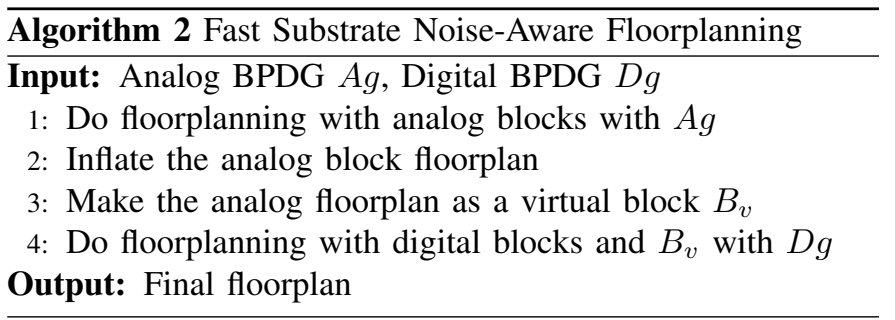

cost functions we used for each algorithm are summarized in Table II. Note that we disabled wirelength optimization, since real implementation of mixed-signal SOCs has sparse interconnection between analog blocks and digital blocks, which is not well reflected in MCNC benchmarks. However, our approach can be readily extended to include wirelength optimization, maintaining high computational efficiency.

All algorithms were tested on a Pentium4 Linux machines with MCNC [16] benchmarks (ami33, ami49) and two randomly generated larger benchmarks (n75 with 75 blocks, n100 with 100 blocks). About $30 \%$ of the blocks in each benchmark were chosen as analog blocks, and noise characteristics of all the blocks were carefully generated. All process dependent parameters were the same as in [5], [14] and [15].

Table II shows experimental results for all benchmarks with three algorithms. Each number in the table is generated by taking the average of numbers obtained over 250 floorplans. The simulated annealing of each floorplanning is scheduled by Parquet, and stopped after the same number of movements for each benchmark. The final noise quantities for all algorithms were computed based on Eqn. (4) for fair comparison.

The last two columns show the overhead of each algorithm in terms of cpu time and area with respect to parq. From the table, parq shows the best area and cpu time (thus, $0 \%$ overhead), but the worst noise for all benchmarks as expected. The cpu time of bpdg is significantly smaller than that of modl for all benchmarks; bpdg is approximately 6080 times faster than modl. The area overhead of bpdg is slightly smaller for the three larger benchmarks as well than 


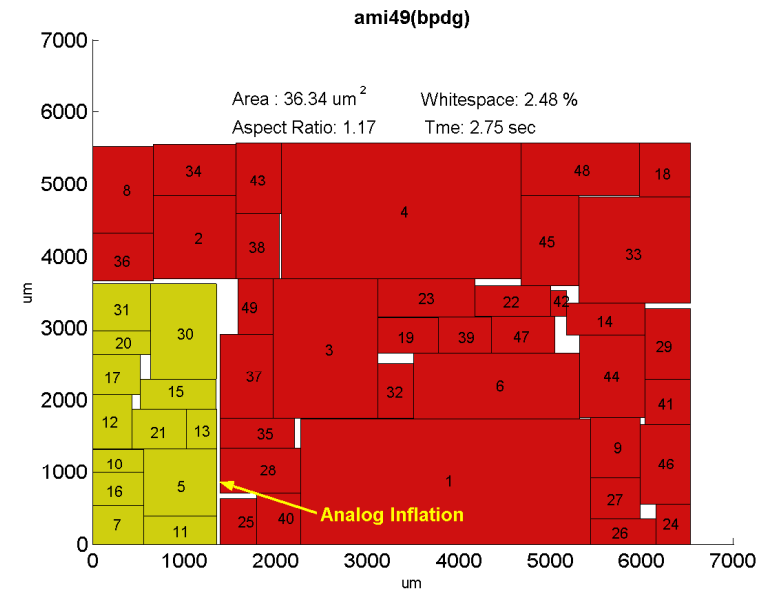

(a) With proposed approach(bpdg)

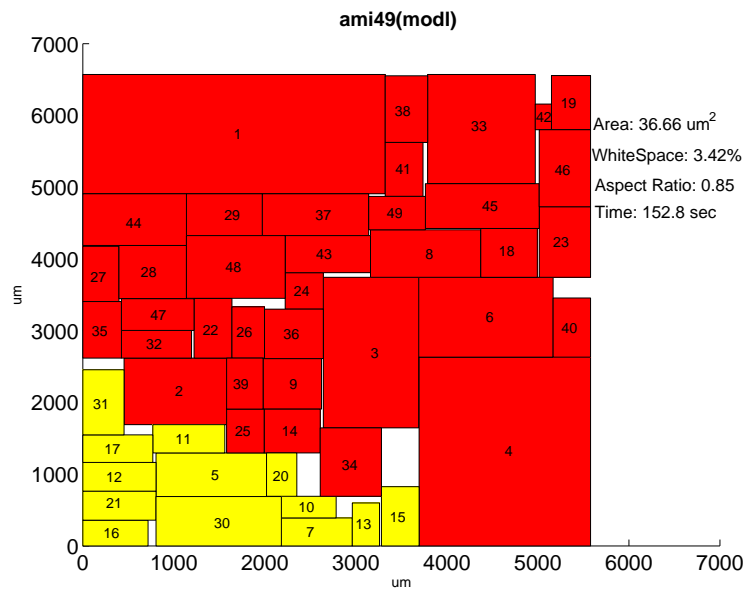

(b) With model-based simulation approach(modl)

Fig. 7. Result of packing ami49

modl. Lastly bpdg shows less total substrate noise than modl. The reason why the proposed algorithm overall shows both smaller area and less substrate noise is that whitespace is more efficiently utilized. By making an analog floorplan inflated as a preprocessing step as in Section $\mathrm{V}$, the substrate noise becomes less in the beginning of annealing, and this allows the simulated annealing engine to optimize the area further without increasing substrate noise. An analogy of this kind of effect can be found in congestion-aware placement [19].

\section{CONCLUSION}

In order to cope with significant substrate noise impact on analog circuits from digital circuits, we propose substrate noise-aware floorplanning with fast substrate noise estimation powered by block preference directed graph (BPDG) and sequence pair. Compared with Parquet [17], the proposed approach has on average only $9 \%$ cpu time overhead, whereas naive model-based simulation approach shows over $6000 \%$ overhead. Also, the proposed approach shows smaller area overhead due to the efficient utilization of whitespace.

Since BPDG is a general concept for fast cost evaluation, it will be extend to deal with temperature or performance estimation in the future.

\section{ACKNOWLEDGEMENT}

The authors would like to thank Prof. Karti Mayaram from Oregon State Univ. and Prof. Ranjit Gharpurey from the Univ. of Texas at Austin for helpful discussions.

\section{REFERENCES}

[1] A. Nardi, H. Zeng, J. L. Garrett, L. Daniel, and A. L. S-Vincentelli, "A Methodology for the computation of an upper bound on noise current spectrum of CMOS swichting activity," in Proc. Int. Conf. on Computer Aided Design, 2003, pp. 778-785.

[2] A. Koukab, K. Banerjee, and M. Declercq, "Modeling Techniques and Verification Methdologies for Substrate Coupling Effects in Mixedsignal System-on-Chip designs," IEEE Trans. on Computer-Aided Design of Integrated Circuits and Systems, vol. 23, no. 6, Jun 2004.
[3] M. V. Heijingen, M. Badarouglu, S. Donnay, G. G. E. Gielen, and H. J. D. Man, "Substrate Noise Generation in Complex Digital systems: efficient modeling and simulation methodology and experiemental verification," IEEE J. Solid-State Circuits, vol. 37, Aug 2002.

[4] H. Lan, Z. Yu, and R. W. Dutton, "A CAD-oriented Modeling Approach of frequency-dependent behavior of Substrate Noise Coupling for Mixed-Signal IC Design," in Proc. Int. Symp. on Quality Electronic Design, Mar 2003, pp. 195-200.

[5] B. Owens, S. Adluri, P. Birrer, R. Shreeve, S. K. Arunachalam, and K. Mayaram, "Simulation and Measurement of Supply and Substrate Noise in Mixed-Signal ICs," IEEE J. Solid-State Circuits, vol. 40, no. 2, Feb 2005.

[6] T. Blalack, Y. Leclercq, and C. P. Yue, "On-chip RF isolation techniques," in Proc. IEEE BCTM., 2002, pp. 205-211.

[7] C. Lin and D. Leenaerts, "A New Efficient Method Substrate-Aware Device-Level Placement," in Proc. Asia and South Pacific Design Automation Conf., 2000, pp. 533-536.

[8] G. Blakiewicz, M. Jeske, M. Chrzanowska-Jeske, and J. S. Zhang, "Substrate Noise Modeling in Early Floorplanning of Mixed-Signal SOCs," in Proc. Asia and South Pacific Design Automation Conf., Jan 2005, pp. 819-823.

[9] H. Murata, K. Fujiyoshi, S. Nakatake, and Y. Kajitani, "VLSI Module Placement Based on Rectangle-Packing by the Sequence-Pair," vol. 15, Dec 1996

[10] X. Tang and D. Wong, "Floorplanning with Alignment and Performance Constraints," in Proc. Design Automation Conf., Jun 2002.

[11] W. K. Chu, N. Verghese, K. S. H. Cho, H. Tsujikawa, S. Hirano, S. Doushoh, M. Nagata, A. Iwata, and T. Ohmoto, "A Substrate Noise Analysis Methodology for Large-Scale Mixed-Signal ICs," in Proc. IEEE Custom Integrated Circuits Conf., 2003.

[12] N. K. Verghese and J. J. Allstot, "Computer-aided design considerations in Mixed-signal coupling in RF integration circuits," IEEE J. Solid-State Circuits, vol. 33, Mar 1998.

[13] J. P. Costa, M. Chou, and L. M. Silveria, "Efficient techniques for accurate modeling and simulation of substrate coupling in Mixed-signal ICs," IEEE Trans. on Computer-Aided Design of Integrated Circuits and Systems, vol. 18, no. 5, pp. 597-607, May 1999.

[14] D. Ozis, T. Fiez, and K. Mayaram, "An Efficient Modeling Approach for Substrate Noise Coupling Analysis," in Proc. IEEE Int. Symp. on Circuits and Systems, 2002.

[15] — - "Comprehensive geometry-dependent macromodel for substrate noise coupling in heavily doped cmos processes," in Proc. IEEE Custom Integrated Circuits Conf., 2002.

[16] http://www.cse.ucsc.edu/research/surf/GSRC/MCNC

[17] S. N. Adya and I. L. Markov, "Fixed-outline Floorplanning : Enabling Hierarchical Design," IEEE Trans. on Very Large Scale Integration (VLSI) Systems, vol. 11, pp. 120-1135, Dec 2003.

[18] http://vlsicad.eecs.umich.edu/BK/parquet.

[19] U. Brenner and A. Rohe, "An effective congestion driven placemnet framework," in Proc. Int. Symp. on Physical Design, 2002, pp. 6-11. 